\title{
Identification and Characterization of DIGI Promoter Involved in Photoperiod, Light Intensity, Hormone, and DIELF4 Response from Longan
}

\author{
Saquib Waheed, Yuan Peng, and Lihui Zeng \\ Institute of Genetics and Breeding in Horticultural Plants, College of Horticulture, Fujian Agriculture \\ and Forestry University, Fuzhou 350002, China
}

\begin{abstract}
AdDitional INDEX words. circadian rhythm, Dimocarpous longan, ELF4, flowering induction, GI
Abstract. In fruit trees, flowering is a key event followed by fruit development and seed production. Gigentea (GI), a clock-associated gene, is known to contribute to photoperiodic flowering and circadian clock control in Arabidopsis thaliana. However, its functions in woody fruit trees remain unclear. In this study, a 2000 bp promoter fragment of the longan (Dimocarpous longan) DIGI gene was isolated from the genomic DNA of longan 'Honghezi' by polymerase chain reaction amplification. The DIGI promoter contained two main types of potential cis-acting elements: lightresponsive and hormone-responsive elements. The promoter was fused with the $\beta$-glucuronidase (GUS) reporter gene of pBI121 to generate the pDIGI:GUS construct. GUS histochemical staining of transgenic $A$. thaliana revealed that $D I G I$ might play a role in different developmental phases of longan. Exposure of transgenic $A$. thaliana to varying light intensities showed that the $G U S$ activity increases with increased light intensity. Transient expression of pDIGI::GUS in Nicotiana benthamiana showed that the $G U S$ activity was higher and reached peak a few hours earlier under shortday (SD) than long-day conditions. Exposure to different hormonal treatments revealed that the transcript level of $G U S$ was activated by gibberellin $\left(\mathrm{GA}_{3}\right)$ and indoleacetic acid (IAA) but suppressed by abscisic acid and methyl jasmonate treatment. In addition, $N$. benthamiana transient assay and dual-luciferase assay revealed that the presence of early flowering 4 (ELF4) homologs of longan (DIELF4-1 and DIELF4-2) significantly activated the DIGI promoter. The positive response of $D I G I$ promoter to high light-intensity, SD photoperiod, GA $\mathrm{A}_{3}$ and IAA signals, and DIELF4 transcription factor suggest that $D I G I$ may function as a circadian clock and play a role in responding to SD conditions and other signals in flower initiation of longan.
\end{abstract}

Dimocarpus longan (longan) is an evergreen fruit tree that belongs to the Sapindaceae family and grows well in many tropical and subtropical regions of the world. China is topranked in terms of the plantation area and fruit production of longan (Matsumoto, 2006; Wu, 2008). Flowering is a crucial developmental phase of plant life, especially in the case of fruit trees, for which the transition of flowering is essential for the fruit set and ripening process (Peng et al., 2008). Generally, longan trees have a single spring flowering period. However, one cultivar of longan, Sijimi, originating in China (Guangxi province), has a perpetual flowering trait and has been successfully used to produce off-season fruit without any additional environmental stimuli (Jia et al., 2014). RNA sequencing results revealed that there were several flowering-time homologs in longan, including gigentea (GI) and early flowering 4 (ELF4), which could be involved in the perpetual flowering traits of 'Sijimi' (Jia et al., 2014). Moreover, our previous studies showed that the longan $G I$ gene $(D l G I)$ has a higher expression level during the floral bud physiological differentiation stage (Huang et al., 2017). DlGI is supposed to be involved in the regulation of physiological differentiation of floral buds, inducing floral initiation by affecting endogenous auxin synthesis and transport in longan (Huang et al., 2017).

Received for publication 13 May 2020. Accepted for publication 16 June 2020. Published online 10 September 2020.

This work was supported by The Construction of Plateau Discipline of Fujian Province (102/71201801101), China.

L.Z. is the corresponding author. E-mail: 1hzeng@fafu.edu.cn or lhzeng@hotmail.com.

This is an open access article distributed under the CC BY-NC-ND license (https://creativecommons.org/licenses/by-nc-nd/4.0/).
Multiple flowering time pathways are controlling the vegetative-to-reproductive phase transition, and $G I$ is proposed to have essential functions in promoting the photoperiodic flowering pathways (Mishra and Panigrahi, 2015). In Arabidopsis thaliana, GI plays a vital role in the regulation of flowering time by controlling the daily endogenous rhythms, also known as circadian rhythms (Dodd et al., 2005). GI was found to induce flowering in long-day (LD) photoperiods by regulating miR172 accumulation (Jung et al., 2007). Plants having a defect in maintaining circadian rhythm usually show altered flowering phenotypes (Imaizumi, 2010), and defects in circadian clock components have been shown to affect $G I$ transcription (Mishra and Panigrahi, 2015). The overexpression of $G I$ exhibited early flowering phenotypes (Mishra and Panigrahi, 2015). While mutation in GI delays flowering under LD conditions (Park et al., 1999), short circadian periods (Mizoguchi et al., 2005), long hypocotyls (Araki and Komeda, 1993), and sensitivity to low-temperature exposures (Cao et al., 2005).

Recent studies demonstrated that internal and external signals affect $G I$ expression in A. thaliana (Berns et al., 2014). The transcription of the GI gene is activated by exposure to light and regulated by the circadian clock (Paltiel et al., 2006; Sawa and Kay, 2011). GI participates in flowering induction by extending photoperiods; therefore, GI is a key component in the mechanism leading to day-length discrimination (Sawa et al., 2007). Mutations within the components of the morning loop, such as CCA1 and LHY, result in GI transcription to occur earlier in the circadian cycle, suggesting that these proteins repress or delays the GI transcription when they are expressed in the morning (Mizoguchi et al., 2002). The CCA1 and LHY bind to several 
motifs that are closely associated in sequence, including "evening element" (EE) (Harmer and Kay, 2005; Mikkelsen and Thomashow, 2009). In addition to light regulation, EE is also found in close proximity of abscisic acid (ABA) ABREL motifs (Mikkelsen and Thomashow, 2009). Berns et al. (2014) suggested a composite effect of ABRELs and $\mathrm{EE}$ that contributes to generating the observed pattern of $G I$ transcription in $A$. thaliana in the evening. ELF4, another essential component of circadian clock input pathways (McWatters et al., 2007). Overexpression of ELF4 shows delayed flowering signs in A. thaliana, while early flowering was observed in ELF4-deficient mutants (McWatters et al., 2007). In A. thaliana, ELF4 has been proposed to interact with $G I$ to control the hypocotyl elongation and flowering time (Kim et al., 2012). ELF4 is epistatic to GI during hypocotyl elongation control, whereas $G I$ is epistatic to ELF4 regarding flowering time regulation (Kim et al., 2012). A study in pea (Pisum sativum) supposed that Late bloomer 1 (LATE1) and Die neutralis (DNE), orthologs of ELF4 and GI, respectively, interact genetically to regulate flowering time (Liew et al., 2009).

Flowering induction in perennial fruit trees is much different from annual plants. Perennial plants sense the seasonal changes and initiate flowering. However, up to now, understanding of the functions of $G I$ homologs in perennial fruit trees have been limited. On the basis of previous research, we speculate that DlGI may play a role in longan physiological differentiation of floral buds by sensing internal and external signals. It would be interesting to investigate the upstream regulatory factors of $D l G I$. Therefore, in this study, the promoter of $D l G I$ gene was cloned, and bioinformatics analysis, $A$. thaliana stable transformation, Nicotiana benthamiana transient cotransformation, and dual luciferase assay were conducted. The results show that $D l G I$ responded to photoperiod, light intensity, hormones, and DlELF4 genes. These findings will contribute to a better understanding of $D l G I$ functions and the molecular mechanism of flowering induction in perennial fruit trees.

\section{Materials and Methods}

Plant Material. The seeds of $N$. benthamiana were grown in pots containing sterilized compost mix and maintained in a growth chamber. Young $N$. benthamiana leaves were used for transient expression. The $A$. thaliana ecotype Columbia (Col-0) seeds were directly grown in plug trays containing compost mix and maintained in a growth chamber at 22 to $25^{\circ} \mathrm{C}, 50 \%$ to $70 \%$ humidity, and a 16-h photoperiod.

ISOLATION OF D $\boldsymbol{L}$ GI PROMOTER AND BIOINFORMATIC ANALYSIS. The DlGI promoter sequence was amplified (for primers, see Supplemental Table 1) from longan genomic DNA that had been extracted from leaf samples using the cetyltrimethylammonium bromide method (Turaki et al., 2017). The polymerase chain reaction (PCR) reaction for $D l G I$ promoter was executed as per the following protocol: initial preheating for $30 \mathrm{~s}$ at $94^{\circ} \mathrm{C} ; 35$ cycles of $94{ }^{\circ} \mathrm{C}$ for $30 \mathrm{~s}, 61{ }^{\circ} \mathrm{C}$ for $90 \mathrm{~s}$, and $72{ }^{\circ} \mathrm{C}$ for $2 \mathrm{~min}$; and a final elongation step of $72^{\circ} \mathrm{C}$ for $5 \mathrm{~min}$. The amplified fragment was cloned into the pMD-18-T vector, and positive colonies were selected at random and sent for sequencing to Biosune, Shanghai, China. PLACE (Higo et al., 1998) and PlantCARE (Lescot et al., 2002) databases were used for predicting the regulatory elements and potential core sequences of $D l G I$ promoter.

Plasmid construction. The $D l G I$ promoter was cloned into the plant expression vector pBI121, which contained GUS as a reporter gene, using an In-Fusion HD cloning kit (Clontech
Laboratories, Beijing, China). The pBI-121 plasmid was digested with restriction endonuclease HindIII and EcoRI. The cut product was recovered by the gel electrophoresis and ligated with the $D l G I$ promoter to obtain a recombinant plasmid pDlGI::GUS. The verified pDlGI::GUS construct was transformed into a Agrobacterium tumefaciens GV3101. The pBI121DlELF4-1 and pCAMBIA2300-DlELF4-2 plasmids (Fu et al., 2018) were also transformed into A. tumefaciens GV3101.

A. THaLIANA TRANSFORMATION. The pDIGI::GUS transgenic $A$. thaliana lines were established using the floral dip method on the development of inflorescence stems, described by Zhang et al. (2006). The putative transformants were selected on Murashige and Skoog (MS) medium with (50 mg. $\left.\mathrm{L}^{-1}\right)$ kanamycin. Cetyltriethylammnonium was used to extract genomic DNA from the leaf tissues of transformed plants (Wang et al., 1996). PCR analysis was performed to verify the transgenic plantlets by using $D l G I$ promoter specific-primers (F: 5' CGCGACTAACATGTATCAATGGTC-3'; R: 5' -CCGCGTATAGGAAAACAAAGAGG-3'). Plants corresponding to the T3 generation were selected for further experiments.

$\boldsymbol{G} \boldsymbol{U} \boldsymbol{S}$ ACTIVITY ASSAY. GUS activity was visualized histochemically using the chromogenic substrate 5-bromo-4-chloro3 -indyle- $\beta$-D-glucuronide (X-Gluc) for staining, according to the method described by Jefferson et al. (1987). Transgenic $A$. thaliana plants were incubated in GUS-staining solution $(2 \mathrm{~mm}$ $\mathrm{K}_{3} \mathrm{Fe}(\mathrm{CN})_{6}, 1 \mathrm{~mm} \mathrm{X}$-Gluc, $\left.2 \mathrm{~mm} \mathrm{~K} \mathrm{Fe}_{4} \mathrm{FN}\right)_{6}, 100 \mathrm{mg} \cdot \mathrm{mL}^{-1}$ chloramphenicol in $50 \mathrm{~mm} \mathrm{NaH}_{2} \mathrm{PO}_{4}$ buffer $(\mathrm{pH} 7.0), 0.1 \%(\mathrm{v} /$ v) Triton X-100; Sigma-Aldrich, St. Louis, MO), for 2 to $3 \mathrm{~d}$ at $37^{\circ} \mathrm{C}$. Plant material was then incubated in $100 \%$ ethanol for 4 to $5 \mathrm{~h}$ to remove chlorophyll from the plant material.

Activity of DLGI promoter in $\boldsymbol{A}$. Thaliana. We selected six independent T2 transgenic lines (genetically unique) for each treatment. The T3 seeds (six lines of each antibioticresistant line) obtained were further selected on MS medium supplemented with $50 \mathrm{mg} \cdot \mathrm{L}^{-1} \mathrm{kanamycin}$ to obtain plants that were homozygous. Ten-day-old T3 resistant seedlings were transferred into the pots containing the compost mix for further expression analysis at lateral developmental stages and incubated them in the GUS substrate as previously described. GUS activity sites were observed by a stereo fluorescence microscope (SZX16; Olympus, Tokyo, Japan).

RESPONSE OF DLGI PROMOTER TO DIFFERENT INTENSITIES OF LIGHT. The 7-d-old T3 transgenic $A$. thaliana plants were kept in growth chambers for $3 \mathrm{~d}$ at $22{ }^{\circ} \mathrm{C}$ under different light intensities, including high light $\left(1100 \mu \mathrm{mol} \cdot \mathrm{m}^{-2} \cdot \mathrm{s}^{-1}\right)$, weak light $\left(400 \mu \mathrm{mol} \cdot \mathrm{m}^{-2} \cdot \mathrm{s}^{-1}\right)$, and dark conditions.

N. BENTHAMIANA TRANSIENT TRANSFORMATION. For photoperiod treatment, $N$. benthamiana plants leaves were infiltrated with $A$. tumefaciens harboring vector pDlGI::GUS and kept either in LD (16 h/8 h light-dark) conditions with the photoperiod starting at $0800 \mathrm{HR}$ and ending at $0000 \mathrm{HR}$, or in $\mathrm{SD}(8 \mathrm{~h} / 16 \mathrm{~h}$ light-dark) with the photoperiod starting at $0800 \mathrm{HR}$ and ending at $1600 \mathrm{HR}$, for $72 \mathrm{~h}$ at $22{ }^{\circ} \mathrm{C}$. To observe the effect of different hormones on the transcriptional activity of $G U S, N$. benthamiana leaves were infiltrated with $A$. tumefaciens harboring pDIGI::GUS construct and sprayed with hormone treatment [34.6 $\mu \mathrm{M}$ gibberellin $\left(\mathrm{GA}_{3}\right), 8.6 \mu \mathrm{M}$ IAA, $75.7 \mu \mathrm{M}$ ABA, $100 \mu \mathrm{M}$ methyl jasmonate (MeJA)] and sterile water for the control.

To investigate the regulation of DlELF4 by DlGI promoter, A. tumefaciens strains containing either of DlELF4 genes or pDIGI::GUS were separately cultured and diluted to measure the absorbance at a wavelength at $\mathrm{A}_{600}$ of 0.6 to 0.8 . 
1930 AAAAGATTTACTACCTGCAATCAAAACTAAATATTACGTATAATTTGAAAATTTATTCACATTATGAAAA

-1860 CAAACTTCTCAAGACATATAAGTACTTATACAACATAGAACTTGAACTTTTATTACAAATACGAAGGATA

-1790 GATATGCTAATTACTTGAATTATGAGCGAATATATAATATCAACATAAAATTTAAAATATATAGAAGCTT AT ${ }^{\sim}$ TATA-box

1720 TACATTTATGTTAAATATAAAAGTCTAAAAACAATACAAAATGAATTATAGTTGg GTTTCTTAACATTTA

-1650 TGGGAAGTACGTCCACTCATATTTCAAGTACCATCTGCATTTATTGGCATATGTGGGAAATTTTTAATTC

-1580 TAATTG TAAAAATAG ATTTTTCTCGATAAAT TTATG AGCATTTATATAAGTAATTTTTTTGGATATATTA (-)WUN-motif

-1510 CATTACAAGTAACATTGTTGGGTCCTCTAGATCAGGCTACTGAGACACGAGTTGGAACG GGAGGGTCTTT $(-)$ Unnamed 4

-1440 GATGgGAGAAAGGTTTCCTAAATTAGGGAGGAGgGACAACCCAAATAAAGAAAAACTAACCACATTTTAG MYB

-1370 TAGTGGCGGATGTGGAACTCTTAGTTCAATAAAATTCAAATTATATCTIAACTGTACCAAAAATTAATTT (-)CAT-box MYb BOX 4

-1300 TAGCAATG ATTTTAGATAAATTIATAAAATTTAAAATGATTAAATAAAAAACTCGTATTATTATTTAATT TATA

-1230 TAAACGAATTATATTTTCTTCCGGAGATGATGAAACAGTTGATCAACAACACGAAGGCGCGGTCTAAAAA GAG-motif $(-) M B S$

-1160 CAAGGTGGACGTACTCGTGAGCATGACAATCCACCAAACTCCCCTCGAAAATATCTTCTGACAGTTTGAT (-)WRE3 Unnamed_4 EE-motif

1090 TATAAAAACATTAAAATATGCACCAACTAAAACTTGCAATAATTCGTCCAATACTTTAAACAACCCATTT -1020 GAAGGGCAACCG $\underline{\text { CGTCATTTCACACGCCCCAATATGTCAGATGCCAATGGATGCGCATGGGCGATGGTAG }}$ CGTCA-motif

-950 TAGGCATGTCATGGAAGCATGGTGGTTTAGGATGACGTGGCACACGGCCTCTAATGTGAGAGGAAAATAT (-)ARE TGACG-motif/ABRE

-880 ATGCCACGTAGAGACAGCGATCAAAGCTCACAGCCCATTTAAAGATCTCGCTCCCAACGCTTCAACCTCT G-box/ABRE4

-810 TAATAAAATTCCAAATAATAATTTATTTTTTCTTGITTCAAAAAAAAAAAAAAAAAAAAAAAAGGGACAA BOX 1

-740 TTGTATTCACAGAAGCAAATATCCAAAAAAACCGAGAGAAGCGGAGACCTGGATTTCTTCTCTGCACCCA DRE1

UTR Py-rich stretch

-670 ACAACTATCAGGTG CTCTGTTTTCTCTCTTCCTCCTTTTTTTTTTTTTTTTTAATTTTTTTATCTTTGAAG

-600 TTCATTTTATTCGTCTATCTTGCTGTTTGCACATTTTTTTGTTAATTATTATCATTATTTTTTTAAACTT

-530 TTAGTTGTTTTGTGTGTGAAGCTAGTGTAAAATCTGTTTTAGCAGTACCTGAAATGAACCTGTTTTTTTA GT1-motif

-460 TTTTGTTTATGGTACTTGCCAATTTATGTGATTTTTATTTTGTTTTTACTTTTGGTTAAATTTCGAATCT GT1-motif

-390 GTGCTGATTTTAGTTTTTTTTTTTTTGATTTATTGTTGGCAGAGTGTTGATATTTTGGGAGTTTGATTGA

-320 GGCTGTTGAGAGGCATAGAGAGAGTAGTGAATTGAATTTTTATTTTTTTTTGGTTITTCGGAGAATTGCA $(-) M Y B$ $(-) L T R$

-250 GCAGCACTAGCAGAAGCAGAAACAGAAACAGAAACAGACTTCGAAATTGTAAAGCTTCATTAATTATTTT CTAG-motif GARE-motif (-)BOX 4

-180 TGAACTGATAATATATACATATTTGGTGAATTCTGGGGGTGGTAGATGTTGGAATGTTGAATTACGAAAT AT TATA-box

-110 TTTGGTTCTTGATTGGTAGCGGTTAGAAAGCGAAAAGAAGGGTTTTTTGATCATTTGAGATTGGAGCTGT

-40 CTGGAAGATATATTCCTCTTTGTTTTCCTATATCA MYC

Fig. 1. Analysis of the DlGI promoter sequence using the PlantCARE database (Lescot et al., 2002).

was evaluated by the $2^{-\Delta \Delta \mathrm{Ct}}$ method (Livak and Schmittgen, 2001).

Dual-luciferase assay. The dual-luciferase assay was carried out according to the method described by Hellens et al. (2005) and Espley et al. (2007). Transient assays were performed on $N$. benthamiana leaves, and plants were kept in a growth chamber for $3 \mathrm{~d}$. Leaf discs of $1 \mathrm{~cm}$ diameter were taken as samples for each treatment, and cell lysate was added into the grinded samples, followed by incubation on ice for $5 \mathrm{~min}$. The firefly luciferase (LUC) and Renilla luciferase (REN) assays were accomplished with the help of a dualluciferase reporter assay kit (Yeasen Biotech Co., Shanghai, China). Luciferase activity was determined with the help of a luciferase assay system, and the setting for fluorescence value measurement reading time was $1000 \mathrm{~ms}$ in the luminometer. The LUC to REN ratio was used to quantify promoter activity.

Statistical analysis. Data are shown as the means with standard errors of three independent biological replicates. The statistical analysis was carried out using a $t$ test and one-way analysis of variance. Least significant difference values were calculated by $P=0.05$ to compare the treatment means. In all graphs, the error bars indicate the standard deviation.

\section{Results}

Cloning of $D_{L} G I$ PROMOTER AND SEQUENCE ANALYSIS. A 2000-bp 5'flanking sequence upstream of the DlGI translation site (ATG) was obtained from longan genomic DNA, covering a previously cloned

A. tumefaciens carrying either DlELF4-1 or DlELF4-2 were mixed separately with a culture carrying pDIGI::GUS at a $1: 1$ ratio, as described by Shamloul et al. (2014), and infiltrated in $N$. benthamiana leaves.

RNA Extraction and Quantitative PCR analysis. The RNA extraction for $N$. benthamiana and A. thaliana were carried out with the help of plant Total RNA Extraction Kit (Biotech Corp., Beijing, China). An ultraviolet spectrophotometer (ND-1000; Nanodrop Technologies, Wilmington, DE) was used to measure RNA concentrations. The cDNA synthesis was carried out via a SMARTer 50/30 kit (Clontech Laboratories). To analyze the expression level of the GUS gene under a series of different treatments, quantitative PCR (qPCR) was conducted using GUS gene specific-primers, and N. benthamiana actin was used as a reference gene (Supplemental Table 2) (David et al., 2006; Jefferson et al., 1987). The relative gene expression
1338 bp 5'-flanking sequence (GenBank: KT429718). Thereafter, the PlantCARE and PLACE databases were used to investigate the regulatory elements and potential core sequences of the DlGI promoter (Supplemental Table 1). Many putative cis-acting elements, including 24 CAAT boxes, which are responsible for the tissue-specific activity of the promoter, and 34 TATA boxes, required for critical and precise transcription initiation, were found dispersed over the entire promoter sequence. Two main types of cis-acting elements were found within the promoter sequence: light- and hormone-responsive regulatory elements (Fig. 1). The light-responsive elements include the box II, Box 4, G-box, G-Box, Box I, GAG-motif, GT1-motif, and EE. Whereas the hormoneresponsive regulatory elements include the auxin-responsive element (TGA-box), the gibberellin-responsive element (GAREmotif), the MeJA-responsive element (CGTCA-motif) and the abscisic acid-responsive element (ABRE) (Fig. 1). 
Stress-related elements were also found in the promoter region, for example, LTR, a cis-acting element involved in low-temperature responsiveness.

SPATIOTEMPORAL EXPRESSION OF THE DLGI PROMOTER IN $\boldsymbol{A}$. THALIANA. To examine the activity of DlGI promoter in T3 transgenic $A$. thaliana, the 2000 -bp promoter fragment driven by the GUS reporter gene was monitored using histochemical staining during different developmental stages of plant growth. Six-day-old transgenic $A$. thaliana seedlings grown on MS medium were strongly stained, and the whole plantlet showed the GUS activity except roots (Fig. 2A). Similarly, GUS activity was observed in 14- d-old plants (Fig. 2B). GUS activity was also detected in the flower buds of 21-d-old transgenic A. thaliana (Fig. 2C). Strong GUS staining was observed in the sepals, petals, and stigma of opened flowers of transgenic $A$. thaliana, but no $G U S$ staining was observed in the anthers (Fig. 2D). GUS staining was also found in the siliques of 40-d-old transgenic A. thaliana (Fig. 2E). As expected, GUS staining was not observed in wild-type $A$. thaliana plants (Fig. 2F).

RESPONSE OF $D_{L} G I$ PROMOTER TO DIFFERENT INTENSITIES OF LIGHT. Bioinformatical analysis of the $D l G I$ promoter sequence revealed that it contains many motifs that are well characterized for their response to light (Table 1), suggesting that different

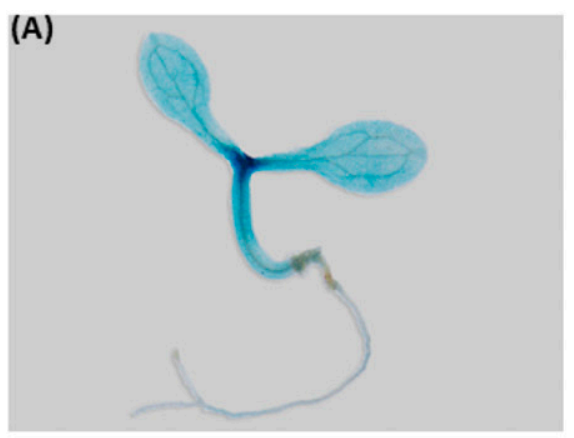

(D)

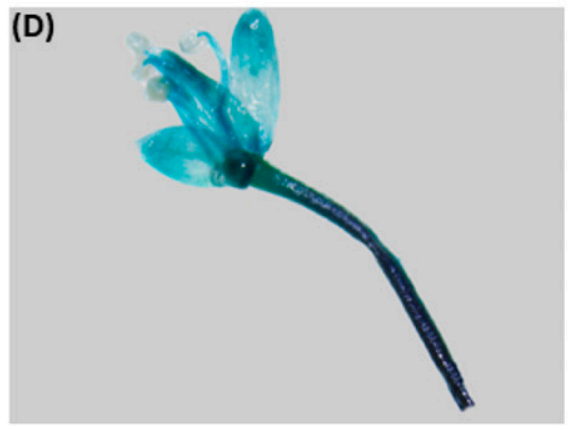

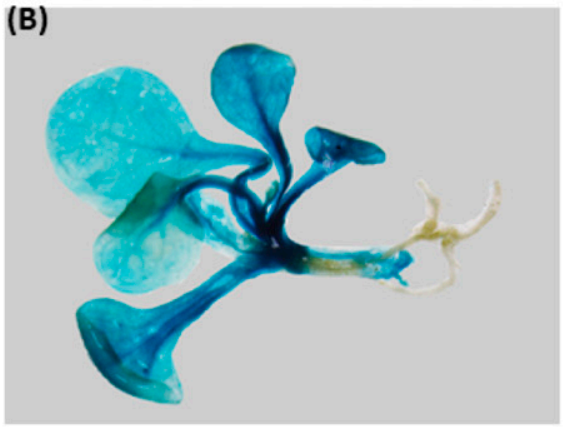

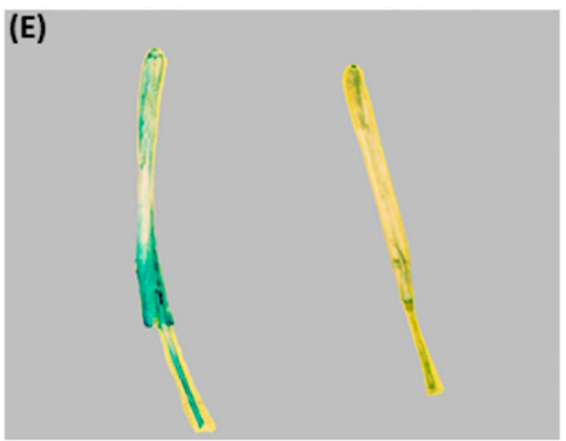

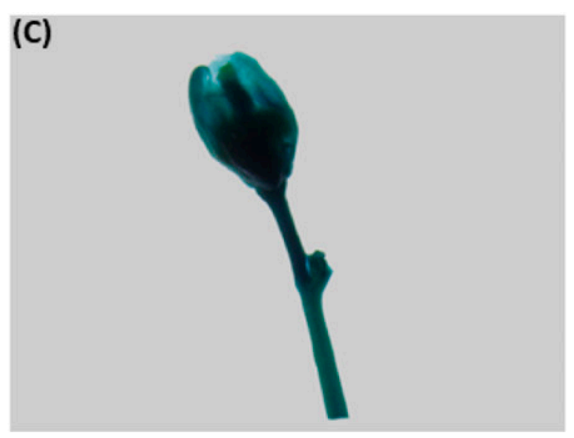

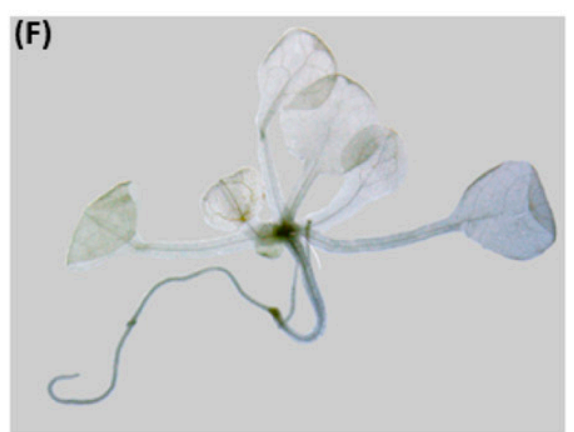

Fig. 2. Histochemical localization of GUS activity in transgenic Arabidopsis thaliana plants carrying the pDlGI::GUS fusion construct: (A) GUS staining localized in the cotyledons of 7-d-old seedlings; (B) 15-d-old plant, showing strong GUS expression in the cotyledons, hypocotyls, and leaves; (C) GUS staining observed in 21-d-old flower bud; (D) GUS staining localized in flowers (sepals, petals, and stigma) of a 26- to 30-d-old plant; (E) 40-d-old plant siliques showing GUS expression; and (F) no expression in wild type A. thaliana.

Table 1. Prediction of regulatory elements in the 5-flanking sequence of DlGI promoter.

\begin{tabular}{|c|c|c|c|}
\hline Regulatory element & Function & No. & Sequence \\
\hline UTR Py-rich stretch & Cis-acting element conferring high transcription levels & 1 & TTTCTTCTCT \\
\hline $\mathrm{ABRE}$ & Cis-acting element involved in the abscisic acid responsiveness & 2 & ACGTG \\
\hline ARE & Cis-acting regulatory element essential for the anaerobic induction & 2 & AAACCA \\
\hline Box I & Light responsive element & 1 & TTTCAAA \\
\hline CGTCA-motif & Cis-acting regulatory element involved in the meja-responsiveness & 3 & CGTCA/CGTCA \\
\hline G-box & Cis-acting regulatory element involved in light responsiveness & 3 & CACGTA \\
\hline GARE-motif & Gibberellin-responsive element & 2 & AAACAGA \\
\hline GT1-motif & Light responsive element & 2 & GTGTGTGAA \\
\hline Sp1 & Light responsive element & 1 & $\mathrm{CC}(\mathrm{G} / \mathrm{A}) \mathrm{CCC}$ \\
\hline LTR & Cis-acting element involved in low-temperature responsiveness & 1 & CCGAAA \\
\hline TGA-box & Part of an auxin-responsive element & 1 & TGACGTGGC \\
\hline box II & Part of a light responsive element & 1 & TCCACGTGGC \\
\hline
\end{tabular}


intensities of light might affect the activity of the $D l G I$ promoter. To validate this hypothesis, pDlGI::GUS activity was measured under different light intensities. Strong GUS activity was detected in seedlings grown under high light intensity, except for the root apex, which did not show any activity (Fig. 3A). Seedlings from reduced light intensity also showed GUS activity in cotyledons and the hypocotyl, but the activity was weaker compared with plants grown in high light intensity (Fig. 3B). Reduced GUS activity appeared in the seedlings grown in dark conditions (Fig. 3C). Wild-type $A$. thaliana was used as a negative control and did not show any $G U S$ activity when grown under a high light intensity (Fig. 3D).

EFFECT OF DIFFERENT PHOTOPERIODS ON DLGI PROMOTER. GI is considered to be an essential clock gene due to its significance in circadian clock regulation. The transient assays were performed to detect the pDIGI::GUS expression under different photoperiods. The results showed that GUS expression was relatively higher in SD conditions compared with LD photoperiods (Fig. 4A). GUS expression was also measured at different points of the day under both LD and SD photoperiod systems. We found that pDIGI::GUS expression peaks at 1200 HR in SD conditions, whereas in LD, the highest expression was observed $\approx 1600$ HR. Under the LD photoperiod system, GUS expression was delayed by a few hours (i.e., the peak was found at a later time point) compared with the SD photoperiod system. Under both photoperiods, the expression levels were lowest in the morning but increased during the day (before dusk) to a peak value and then decreased to a minimum during the dark period (Fig. 4B). These results depict that variation in daily rhythms and photoperiods has definite effects on the expression of $D l G I$.

EFFECT OF DIFFERENT HORMONES ON $D_{L} G I$ PROMOTER. Inspection of the DlGI promoter sequence revealed that it contains a few cis-acting elements that are responsive to hormones, such as gibberellin (GARE-motif), auxin (TGA-box), ABA (ABRE), and MeJA (CGTCA-motif). We performed qPCR to detect the pDlGI::GUS expression under different hormonal treatments. $\mathrm{GA}_{3}$ showed the highest GUS expression compared with other hormone treatments, with a 3-fold higher change than the control. GUS expression was also induced by IAA, with an up to 1.5-fold higher change compared with the control (Fig. 5). In contrast, GUS expression was repressed by ABA and MeJA (Fig. 4).

DLELF4 INCREASES THE TRANSCRIPTIONAL ACTIVITY OF $D_{\boldsymbol{L}} \boldsymbol{G I}$ PROMOTER. The ELF4 gene is known to be involved in many of the same physiological processes as GI. We examined whether there is any direct interaction between DlELF4 and DlGI. Two DlELF4 genes, DlELF4-1 and DlELF4-2, were injected with pDlGI::GUS, respectively, and $G U S$ expression was assayed by qPCR. Results showed that both DlELF4 genes significantly increased the transcriptional activity of the $D l G I$ promoter, especially DlELF4-2 (Fig. 6A). Furthermore, a dual luciferase assay was performed to verify the interaction between $D l G I$ promoter and DlELF4. The results were consistent with the
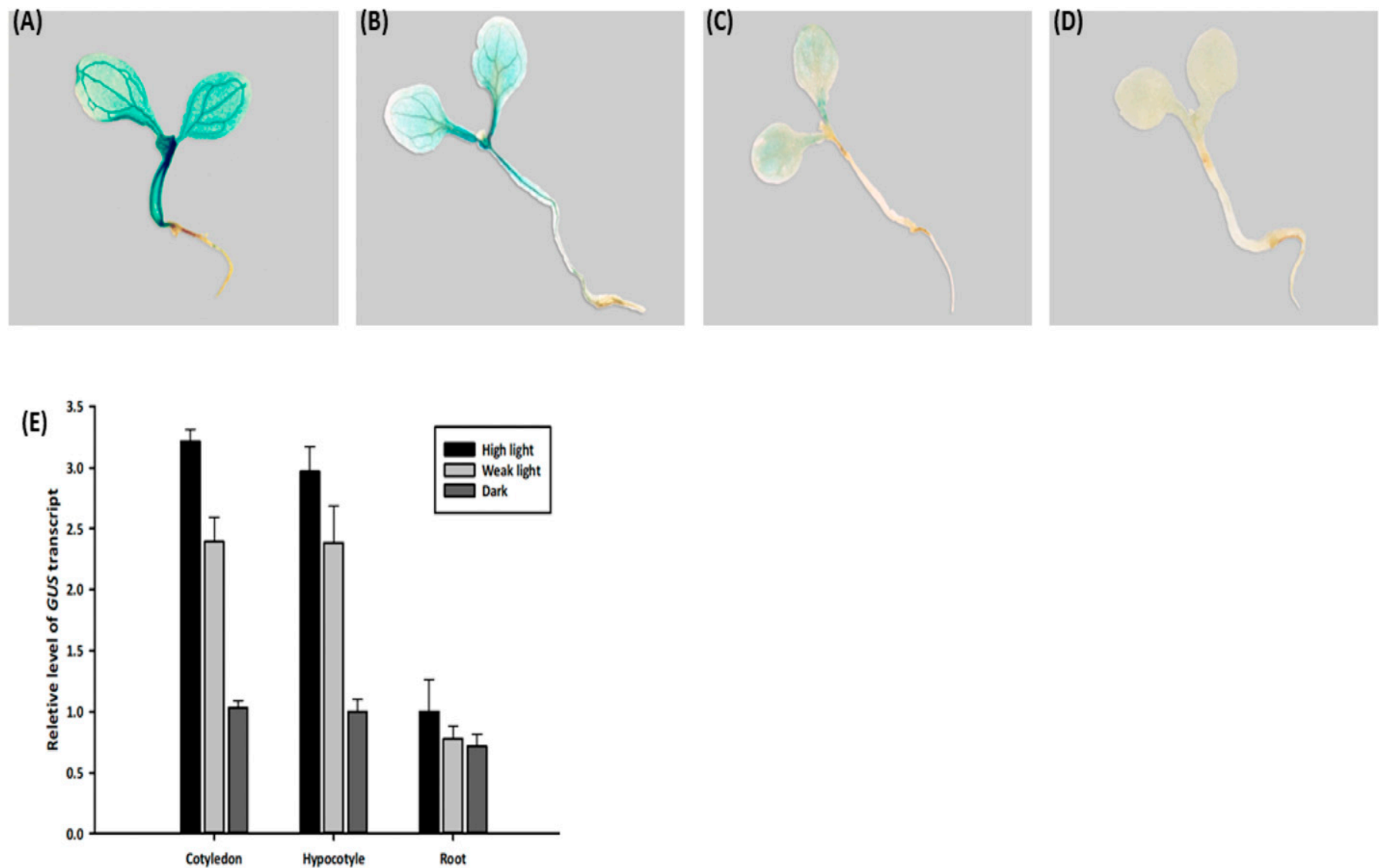

Fig. 3. GUS expression in transgenic Arabidopsis thaliana seedlings under different light conditions: (A) high light $\left(1100 \mu \mathrm{mol} \cdot \mathrm{m}^{-2} \cdot \mathrm{s}^{-1}\right)$; $(\mathbf{B}) \mathrm{weak}$ light $(400$ $\mu \mathrm{mol} \cdot \mathrm{m}^{-2} \cdot \mathrm{s}^{-1}$ ); (C) dark conditions; (D) GUS staining in wild type A. thaliana (negative control); (E) level of GUS transcript in cotyledon, hypocotyl, and root under different intensity of light. Bars indicate the SE. 


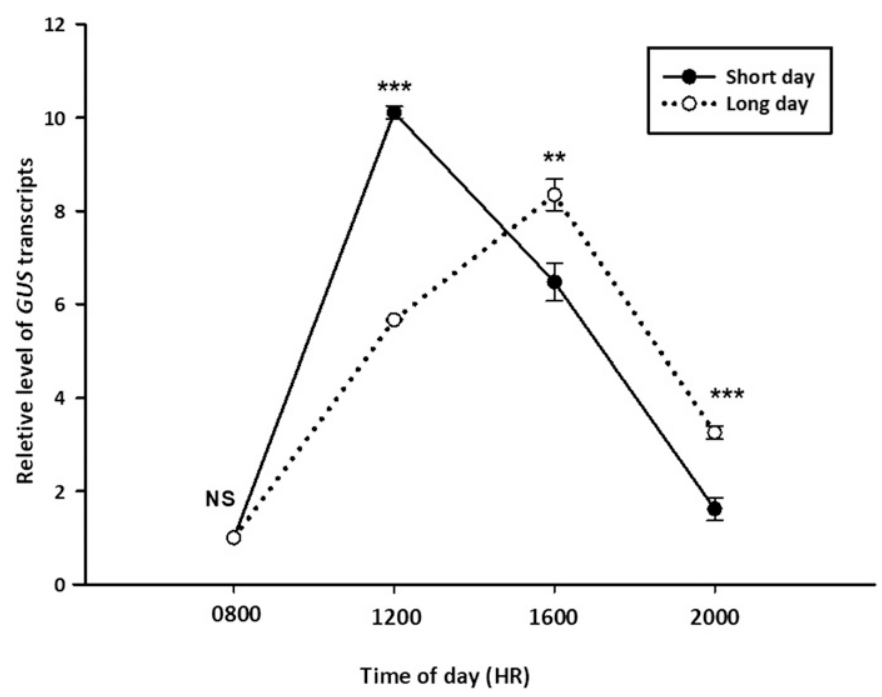

Fig. 4. Effect of different photoperiod on the activity of $D l G I$ promoter infiltrated in Nicotiana benthamiana leaves: (A) DlGI promoter activity under long day ( $16 / 8 \mathrm{~h}$ light/dark) and short day ( $8 / 16 \mathrm{~h}$ light/dark) photoperiod; (B) DlGI promoter activity measured at different points of the day. The bars indicate the SE of three biological replicates. The level of significant differences is indicated with an asterisk $\left({ }^{*}\right)$ and were assessed by $t$ test $\left({ }^{*} P<\right.$ $0.05, * * P<0.01$, or $* * * P<0.001)$

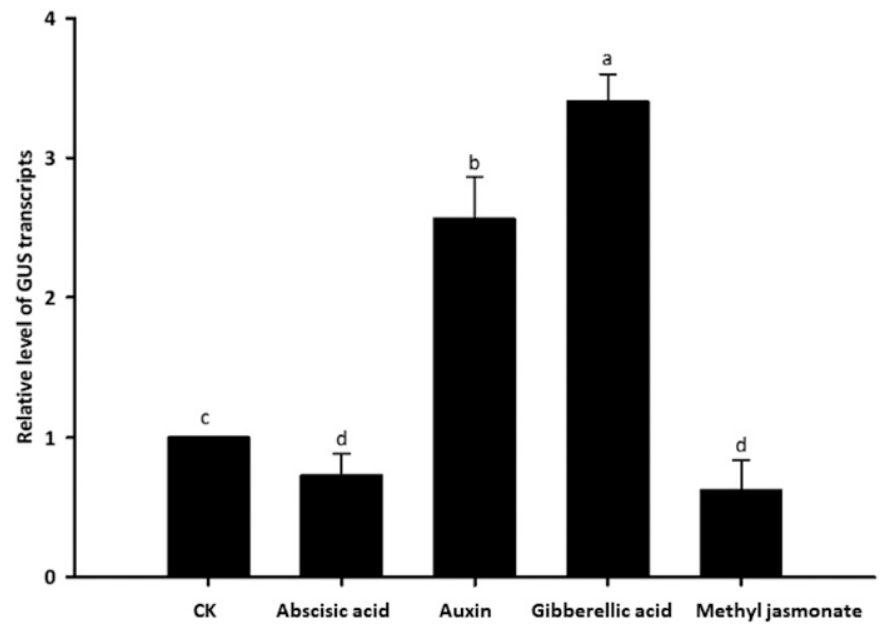

Fig. 5. Response of the DlGI promoter to different hormones. Leaves of Nicotiana benthamiana were infiltrated with the construct pDlGI::GUS were under the treatments of $8.6 \mu \mathrm{M}$ auxin, $34.6 \mu \mathrm{M}$ gibberellin, $100 \mu \mathrm{M}$ methyl jasmonate, $75.7 \mu \mathrm{M}$ abscisic acid, and water, as a control (CK). Bars indicate the SE of three biological replicates. Letters represents a significant difference at the level of $P<0.05$ using least significant difference.

GUS activity assay, showing that both DlELF4 genes increase DlGI promoter activity: DlElF4-2 induced 5-fold higher activity and DlElF4-1 induced 3-fold higher luciferase activity than the control (Fig. 6B).

\section{Discussion}

The plant clock uses daily rhythms and recognizes photoperiods in various developmental processes, from seedling growth to flowering (Kim et al., 2012). The expression profile of the $D l G I$ promoter in transgenic $A$. thaliana suggested that
(A)

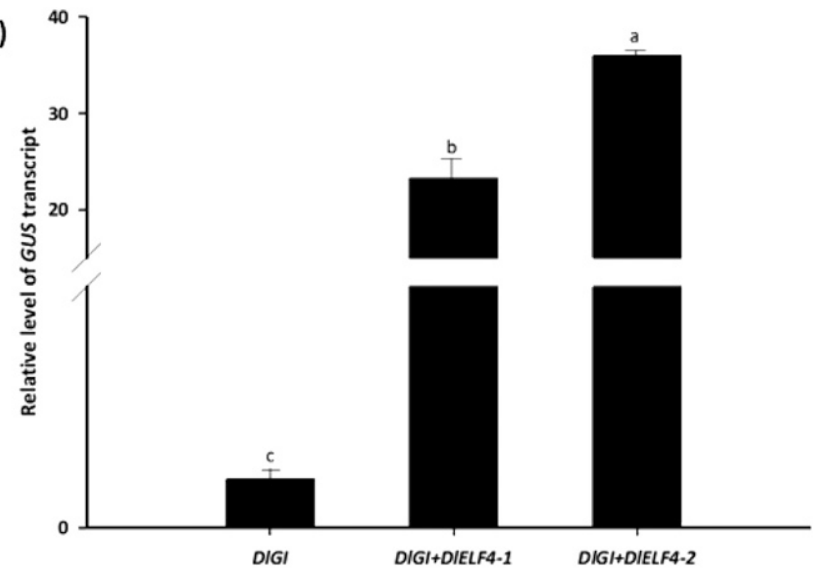

(B)

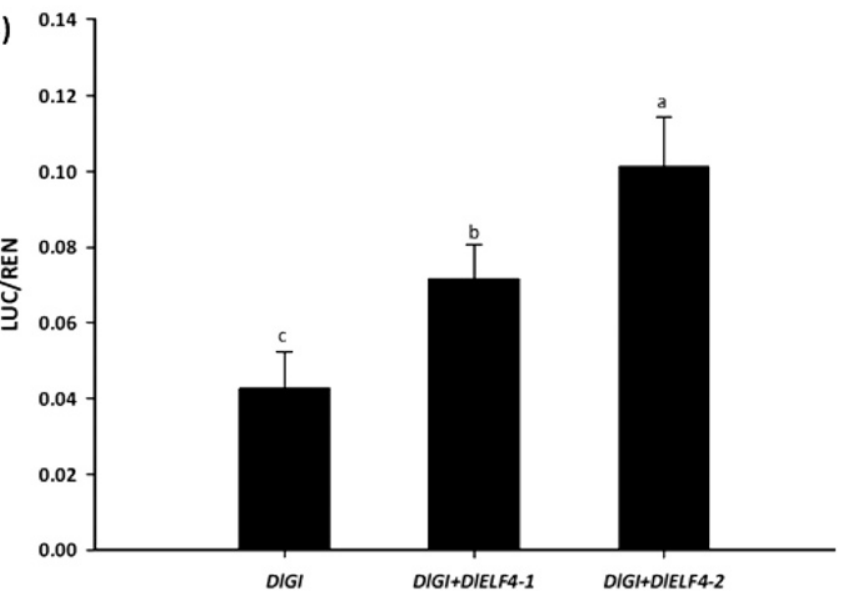

Fig. 6. DlELF4 increase the transcriptional activity of $D l G I$ promoter: (A) pDIGI::GUS coinfiltrated with DlELF4-1 and DlELF4-2 separately in Nicotiana benthamiana leaves. (B) The firefly luciferase (LUC) and Renilla luciferase (REN) assay to study the interaction between $D l G I$ promoter and DlELF4 genes. The bars indicate the sE of three biological replicates. Letter represents a significant difference at the level of $P<0.05$ using least significant difference statistical analysis.

$D l G I$ might play a broader role in different organs and developmental phases of plant growth in longan. A similar kind of $G I$ expression pattern has been observed in A. thaliana (David et al., 2006), Oryza sativa (Hayama et al., 2002), and Glycine max (Li et al., 2013), suggesting DlGI acts as a circadian clock in longan. However, in contrast to GI in A. thaliana, DlGI has a higher expression level in SD conditions, and the expression level reaches its peak earlier in the day under SD conditions compared with LD, suggesting that the expression levels of $D l G I$ are greatly affected by SD-length. Huang et al. (2017) found that $D l G I$ might be involved in the regulation of the physiological differentiation of floral buds. The initiation of flowering in longan occurs during autumn in China, when the photoperiod becomes shorter; therefore, the promoter of $D l G I$ has higher activity under SD conditions, suggesting that $D l G I$ may play a role in responding to SD conditions and inducing flower initiation in longan. Variation in GUS activity under different photoperiods indicates that the conserved motif presented upstream of $D l G I$ may contribute to maximizing the expression at a precise time of the day, thereby exerting control over flowering time. The EE motif present in the promoter represses the transcription of GI during the morning 
and increases the amplitude of expression in the evening (Berns et al., 2014). The ABRELs are predicted to be recognized by bZIP transcription factors, several of which are implicated in light induction of gene transcription (Chattopadhyay et al., 1998). Thus, the contribution of such elements provides a clue in controlling the distinct subsets of a gene by function, possibly due to differing requirements at different times in the day-night cycle.

Light is a well-known environmental factor that plays a substantial role in controlling flowering time in different plants through applied photoperiod, which depends upon the light quality (wavelength) and intensity (Cerdán and Chory, 2003; Lu et al., 2017). Early flowering was observed in 11 herbaceous plant species when they were subjected for $18 \mathrm{~h}$ daily to highintensity lamps, in contrast to plants grown in an ambient environment (Erwin and Warner, 2000). Light quality has a significant role in flower bud initiation and the transition from vegetative to reproductive state in many fruit crops (Wilkie et al., 2008). Several studies in A. thaliana have revealed that the light quantity and quality affect the $G I$ transcription (Fowler et al., 1999; Mishra and Panigrahi, 2015). Our results showed that pDIGI::GUS activity was predominantly induced by highintensity light, compared with low light and dark conditions (Fig. 3A-C), suggesting the expression level of $D l G I$ is upregulated by high light intensity. Recent research on fruit crop flowering has indicated that clock genes involved in flower initiation may generally be mediated by both light quality and quantity (Jackson et al., 2011). Flower bud formation in Malus $\times$ domestica is affected by different intensities of light, as fewer flower buds were formed when the light intensity was reduced for 7 weeks (Tromp, 1983). However, the relationship between light intensity and flowering remains unknown in longan and needs further investigation. Light-responsive cis-elements upstream of DlGI may play crucial roles in the response of GUS gene expression in the transgenic seedlings. Studies related to promoters of light-regulated genes have revealed the presence of different LREs, such as GATA, GT1, and G-box, which play vital roles in light-regulated transcription activity (Yadav et al., 2002). The two most common light-responsive cis-elements in the promoter sequence of $D l G I$ were the G-box, also found in the promoter of photosynthesis-related genes involved in light regulation (López-Ochoa et al., 2007), and the GT-1 motif, which is involved in the regulation of light responses and is usually found clustered with I-box (Escobar et al., 2004).

Some reports have suggested that auxin plays an essential role in the circadian rhythm of flowering stem growth (Jouve et al., 1999). This is demonstrated by the fact that auxin response is linked to processes controlled by the circadian clock and that some circadian clock functions can be influenced by hormone signals (Covington and Harmer, 2007). In this study, $\mathrm{GA}_{3}$ and IAA were found to be upregulated and enhanced the transcriptional activity of GUS. The gibberellin class of phytohormones is involved in the control of flowering in many species (Hedden, 2017). GA 3 is linked with other floral pathways via $\mathrm{GA}_{3}$-regulated $D E L L A$ proteins, creating versatile interacting modules for signaling proteins (Conti, 2017). In longan, the concentration of some hormones, such as IAA, was increased during the floral bud physiological differentiation stage (Yimei, 1997). DlGI expression was also found to be upregulated at the same stage (Huang et al., 2017). The time of change in the concentration of hormones and the change in expression of DlGI coincides with the time when terminal shoots begin flower bud initiation in longan. At the same time, $D l G I$ was predicted to induce floral initiation by affecting endogenous auxin synthesis and transport in longan (Huang et al., 2017); therefore, there may be an IAA-DlGI regulatory loop in longan during flowering induction. However, this hypothesis requires more concrete evidence. Recent studies show that in $A$. thaliana, exogenous application of ABA has exhibited late-flowering phenotype (Achard et al., 2004), also found to participate in circadian clock function and flowering time control (Hanano et al., 2006). Early-flowering phenotypes have been observed in ABA-deficient mutants, showing that ABA acts as an inhibitor of floral transition (Barrero et al., 2005). MeJA was found to inhibit the flowering of short-day plants such as Chenopodium rubrum (Albrechtova and Ullmann, 1994). Pharbitis nil seedlings placed in a solution of MeJA for a period of $24 \mathrm{~h}$ led to a dramatic reduction in the number of formed flower buds (Maciejewska and Kopcewicz, 2002). In this study, abscisic acid and methyl jasmonate were found to reduce the transcriptional activity of the GUS, indicating that they might be inhibitors of flowering in longan.

GI and ELF4 have a similar circadian phase, and they also interact genetically, resulting in identical circadian outcomes in plant growth and flowering time (Kim et al., 2012). Kolmos et al. (2009) found that ELF4 suppresses GI transcription during circadian clock regulation in $A$. thaliana. Jia et al. (2014) demonstrated that the expression level of DlELF4 (UniGene 4309) was higher in 'Sijimi' compared with 'Lidongben,' which suggests that ELF4 may be an essential gene involved in perpetual flowering traits in longan. The data presented in this study demonstrate that DlELF4 genes significantly increase the activity of the $D l G I$ promoter (Fig. 6A and B), suggesting that DlELF4 works upstream of DlGI and promotes $D l G I$ expression (Jia et al., 2014). DlELF4 seems to have different functions from ELF4 in A. thaliana, which is known to destabilize $G I$ protein and be a repressor of flowering. It was reported by $\mathrm{Fu}$ et al. (2018) that the overexpression of longan DIELF4 genes in A. thaliana exhibited late flowering and adventitious root phenotypes, suggesting that DlELF4 genes, and in particular D $l E L F-2$, may regulate hormone biosynthesis. Therefore, DIELF4 may regulate hormone biosynthesis by upregulating $D l G I$ and could be involved in longan flower initiation. However, this hypothesis needs further study. Taken together, the DlGI-DlELF4 interaction provides an additional novel mechanism that can contribute to the currently known genetic and molecular interactions which control photoperiodic flowering.

\section{Conclusions}

In this study, the GI homolog gene was identified as a circadian rhythms gene in the woody fruit tree for the first time. Longan DlGI responds to external changes in photoperiod and light intensity, activated by SD conditions and high light intensity. In addition, DlGI responds to hormones such as $\mathrm{GA}_{3}$, and IAA, and there may be an IAA-DlGI regulatory loop during flowering induction in longan. Moreover, DlELF4 was found to be working upstream of $D l G I$ and activating $D l G I$ expression. The DlELF4-DlGI interaction may provide an additional novel mechanism to the currently known genetic and molecular interactions that control photoperiodic flowering. Our findings not only increase our understanding regarding the upstream regulatory network of $D l G I$ (Supplemental Fig. 1) 
but also provide more knowledge of molecular mechanisms of flowering induction in woody fruit trees.

\section{Literature Cited}

Achard, P., A. Herr, D.C. Baulcombe, and N.P. Harberd. 2004. Modulation of floral development by a gibberellin-regulated microRNA. Development 131:3357-3365.

Albrechtova, J. and J. Ullmann. 1994. Methyl jasmonate inhibits growth and flowering in Chenopodium rubrum. Biol. Plant. 36:317. Araki, T. and Y. Komeda. 1993. Analysis of the role of the lateflowering locus, GI, in the flowering of Arabidopsis. Plant J. 3:231239.

Barrero, J.M., P. Piqueras, M. González-Guzmán, R. Serrano, P.L. Rodríguez, M.R. Ponce, and J.L. Micol. 2005. A mutational analysis of the ABA1 gene of Arabidopsis highlights the involvement of ABA in vegetative development. J. Expt. Bot. 56:2071-2083.

Berns, M.C., K. Nordström, F. Cremer, R. Tóth, M. Hartke, S. Simon, J.R. Klasen, I. Bürstel, and G. Coupland. 2014. Evening expression of Arabidopsis GIGANTEA is controlled by combinatorial interactions among evolutionarily conserved regulatory motifs. Plant Cell 26:3999-4018.

Cao, S., M. Ye, and S. Jiang. 2005. Involvement of GIGANTEA gene in the regulation of the cold stress response in Arabidopsis. Plant Cell Rep. 24:683-690.

Cerdán, P.D. and J. Chory. 2003. Regulation of flowering time by light quality. Nature 423:881-885.

Chattopadhyay, S., L.-H. Ang, P. Puente, X.-W. Deng, and N. Wei. 1998. Arabidopsis bZIP protein HY5 directly interacts with lightresponsive promoters in mediating light control of gene expression. Plant Cell 10:673-683.

Conti, L. 2017. Hormonal control of the floral transition: Can one catch them all? Dev. Biol. 430:288-301.

Covington, M.F. and S.L. Harmer. 2007. The circadian clock regulates auxin signaling and responses in Arabidopsis. PLoS Biol. 5:e222.

David, K.M., U. Armbruster, N. Tama, and J. Putterill. 2006. Arabidopsis GIGANTEA protein is post-transcriptionally regulated by light and dark. FEBS Lett. 580:1193-1197.

Dodd, A.N., N. Salathia, A. Hall, E. Kévei, R. Tóth, F. Nagy, J.M. Hibberd, A.J. Millar, and A.A. Webb. 2005. Plant circadian clocks increase photosynthesis, growth, survival, and competitive advantage. Science 309:630-633.

Erwin, J.E. and R. Warner. 2000. Determination of photoperiodic response group and effect of supplemental irradiance on flowering of several bedding plant species. Acta Hort. 580:95-99.

Escobar, M.A., K.A. Franklin, A.S. Svensson, M.G. Salter, G.C. Whitelam, and A.G. Rasmusson. 2004. Light regulation of the Arabidopsisrespiratory chain. Multiple discrete photoreceptor responses contribute to induction of type II NAD (P) H dehydrogenase genes. Plant Physiol. 136:2710-2721.

Espley, R.V., R.P. Hellens, J. Putterill, D.E. Stevenson, S. KuttyAmma, and A.C. Allan. 2007. Red colouration in apple fruit is due to the activity of the MYB transcription factor, MdMYB10. Plant J. 49:414-427.

Fowler, S., K. Lee, H. Onouchi, A. Samach, K. Richardson, B. Morris, G. Coupland, and J. Putterill. 1999. GIGANTEA: A circadian clockcontrolled gene that regulates photoperiodic flowering in Arabidopsisand encodes a protein with several possible membrane-spanning domains. EMBO J. 18:4679-4688.

Fu, Z., T. Jia, Y. Peng, S. Weheed, and L. Zeng. 2018. Cloning and functional analysis of ELF4 homolog genes in Dimocarpus longan. Acta Hort. Sinica 45:875-886.

Hanano, S., M.A. Domagalska, F. Nagy, and S.J. Davis. 2006. Multiple phytohormones influence distinct parameters of the plant circadian clock. Genes Cells 11:1381-1392.

Harmer, S.L. and S.A. Kay. 2005. Positive and negative factors confer phase-specific circadian regulation of transcription in Arabidopsis. Plant Cell Online. 17:1926-1940.
Hayama, R., T. Izawa, and K. Shimamoto. 2002. Isolation of rice genes possibly involved in the photoperiodic control of flowering by a fluorescent differential display method. Plant Cell Physiol. 43:494-504.

Hedden, P. 2017. Gibberellins, p. 411-420. In: B. Thomas, D.J. Murphy, and B.G. Murray (eds.). Encyclopedia of applied plant sciences. 2nd ed. Academic Press, New York, NY.

Hellens, R.P., A.C. Allan, E.N. Friel, K. Bolitho, K. Grafton, M.D. Templeton, S. Karunairetnam, A.P. Gleave, and W.A. Laing. 2005. Transient expression vectors for functional genomics, quantification of promoter activity and RNA silencing in plants. Plant Methods $1: 13$.

Higo, K., Y. Ugawa, M. Iwamoto, and H. Higo. 1998. PLACE: A database of plant cis-acting regulatory DNA elements. Nucleic Acids Res. 26:358-359.

Huang, F., Z. Fu, L. Zeng, and M. Morley-Bunker. 2017. Isolation and characterization of GI and FKF1 homologous genes in the subtropical fruit tree Dimocarpus longan. Mol. Breed. 37:90.

Imaizumi, T. 2010. Arabidopsis circadian clock and photoperiodism: Time to think about location. Plant Biol. 13:83-89.

Jackson, D., N. Looney, M. Morley-Bunker, and G. Thiele. 2011. Temperate and sub-tropical fruit production. 3rd ed. CABI, Wallingford, UK.

Jefferson, R.A., T.A. Kavanagh, and M.W. Bevan. 1987. GUS fusions: Beta-glucuronidase as a sensitive and versatile gene fusion marker in higher plants. EMBO J. 6:3901-3907.

Jia, T., D. Wei, S. Meng, A.C. Allan, and L. Zeng. 2014. Identification of regulatory genes implicated in continuous flowering of longan (Dimocarpus longan L.). PLoS One 9:e114568.

Jouve, L., T. Gaspar, C. Kevers, H. Greppin, and R. Degli Agosti. 1999. Involvement of indole-3-acetic acid in the circadian growth of the first internode of Arabidopsis. Planta 209:136-142.

Jung, J.-H., Y.-H. Seo, P.J. Seo, J.L. Reyes, J. Yun, N.-H. Chua, and C.M. Park. 2007. The GIGANTEA-regulated microRNA172 mediates photoperiodic flowering independent of CONSTANS in Arabidopsis. Plant Cell Online 19:2736-2748.

Kim, Y., M. Yeom, H. Kim, J. Lim, H.J. Koo, D. Hwang, D. Somers, and H.G. Nam. 2012. GIGANTEA and EARLY FLOWERING 4 in Arabidopsis exhibit differential phase-specific genetic influences over a diurnal cycle. Mol. Plant 5:678-687.

Kolmos, E., M. Nowak, M. Werner, K. Fischer, G. Schwarz, S. Mathews, H. Schoof, F. Nagy, J.M. Bujnicki, and S.J. Davis. 2009. Integrating ELF4 into the circadian system through combined structural and functional studies. HFSP J. 3:350366.

Lescot, M., P. Déhais, G. Thijs, K. Marchal, Y. Moreau, Y. Peer, and S. Rombauts. 2002. PlantCARE, a database of plant cis-acting regulatory elements and a portal to tools for in silico analysis of promoter sequences. Nucleic Acids Res. 30:325-327.

Li, F., X. Zhang, R. Hu, F. Wu, J. Ma, Y. Meng, and Y. Fu. 2013. Identification and molecular characterization of FKF1 and GI homologous genes in soybean. PLoS One 8:e79036.

Liew, L.C., V. Hecht, R.E. Laurie, C.L. Knowles, J.K. Vander Schoor, R.C. Macknight, and J.L. Weller. 2009. DIE NEUTRALIS and LATE BLOOMER 1 contribute to regulation of the pea circadian clock. Plant Cell 21:3198-3211.

Livak, K.J. and T.D. Schmittgen. 2001. Analysis of relative gene expression data using real-time quantitative PCR and the $2^{-\Delta \Delta C T}$ method. Methods 25:402-408.

López-Ochoa, L., G. Acevedo-Hernández, A. Martínez-Hernández, G. Argüello-Astorga, and L. Herrera-Estrella. 2007. Structural relationships between diverse cis-acting elements are critical for the functional properties of a rbcS minimal light regulatory unit. J. Expt. Bot. 58:4397-4406.

Lu, C., S. Zhao, G. Wei, H. Zhao, and Q. Qu. 2017. Functional regulation of ginsenoside biosynthesis by RNA interferences of a UDP-glycosyltransferase gene in Panax ginseng and Panax quinquefolius. Plant Physiol. 111:67-76. 
Maciejewska, B. and J. Kopcewicz. 2002. Inhibitory effect of methyl jasmonate on flowering and elongation growth in Pharbitis nil. J. Plant Growth Regul. 21:216-223.

Matsumoto, T.K. 2006. Genes uniquely expressed in vegetative and potassium chlorate induced floral buds of Dimocarpus longan. Plant Sci. 170:500-510.

McWatters, H.G., E. Kolmos, A. Hall, M.R. Doyle, R.M. Amasino, P. Gyula, F. Nagy, A.J. Millar, and S.J. Davis. 2007. ELF4 is required for oscillatory properties of the circadian clock. Plant Physiol. 144:391-401.

Mikkelsen, M.D. and M.F. Thomashow. 2009. A role for circadian evening elements in cold-regulated gene expression in Arabidopsis. Plant J. 60:328-339.

Mishra, P. and K.C. Panigrahi. 2015. GIGANTEA — an emerging story. Front. Plant Sci. 6:8.

Mizoguchi, T., K. Wheatley, Y. Hanzawa, L. Wright, M. Mizoguchi, H.-R. Song, I.A. Carré, and G. Coupland. 2002. LHY and CCA1 are partially redundant genes required to maintain circadian rhythms in Arabidopsis. Dev. Cell 2:629-641.

Mizoguchi, T., L. Wright, S. Fujiwara, F. Cremer, K. Lee, H. Onouchi, A. Mouradov, S. Fowler, H. Kamada, and J. Putterill. 2005. Distinct roles of GIGANTEA in promoting flowering and regulating circadian rhythms in Arabidopsis. Plant Cell 17:2255-2270.

Paltiel, J., R. Amin, A. Gover, N. Ori, and A. Samach. 2006. Novel roles for GIGANTEA revealed under environmental conditions that modify its expression in Arabidopsis and Medicago truncatula. Planta 224:1255-1268.

Park, D.H., D.E. Somers, Y.S. Kim, Y.H. Choy, H.K. Lim, M.S. Soh, H.J. Kim, S.A. Kay, and H.G. Nam. 1999. Control of circadian rhythms and photoperiodic flowering by the Arabidopsis GIGANTEA gene. Science 285:1579-1582.

Peng, J., L. Xie, B. Xu, J. Dang, Y. Li, Z. Lu, S. Zhang, Z. Yu, X. Bai, and Z. Cai. 2008. Study on Biological Characters of 'Sijihua'. Longan. Acta Hort. 863:249-258.
Sawa, M. and S.A. Kay. 2011. GIGANTEA directly activates Flowering Locus T in Arabidopsis. Proc. Natl. Acad. Sci. USA 108:1169811703

Sawa, M., D.A. Nusinow, S.A. Kay, and T. Imaizumi. 2007. FKF1 and GIGANTEA complex formation is required for day-length measurement in Arabidopsis. Science 318:261-265.

Shamloul, M., J. Trusa, V. Mett, and V. Yusibov. 2014. Optimization and utilization of Agrobacterium-mediated transient protein production in tobacco. J. Vis. Exp. 86:e51204.

Tromp, J. 1983. Flower-bud formation in apple as affected by air and root temperature, air humidity, light intensity, and day length. Acta Hort. 149:39-48.

Turaki, A., B. Ahmad, U. Magaji, U. Abdulrazak, B. Yusuf, and A.B. Hamza. 2017. Optimised cetyltrimethylammonium bromide (CTAB) DNA extraction method of plant leaf with high polysaccharide and polyphenolic compounds for downstream reliable molecular analyses. Afr. J. Biotechnol. 16:1354-1365.

Wang, X.-D., Z.-P. Wang, and Y.P. Zou. 1996. An improved procedure for the isolation of nuclear DNA from leaves of wild grapevine dried with silica gel. Plant Mol. Biol. Rpt. 14:369-373.

Wilkie, J.D., M. Sedgley, and T. Olesen. 2008. Regulation of floral initiation in horticultural trees. J. Expt. Bot. 59:3215-3228.

$\mathrm{Wu}, \mathrm{S}$. 2008. Production status of longan in China. Acta Hort. $863: 37-44$

Yadav, V., S. Kundu, D. Chattopadhyay, P. Negi, N. Wei, X.W. Deng, and S. Chattopadhyay. 2002. Light regulated modulation of Z-box containing promoters by photoreceptors and downstream regulatory components, COP1 and HY5, in Arabidopsis. Plant J. 31:741-753.

Yimei, S.M.L.Z.Z. 1997. Effects of endogenous hormones in bearing basal shoots of 'Shuizhang' longan on floral bud differentiation. Chinese J. Trop. Crop. 18:66-71.

Zhang, X., R. Henriques, S.-S. Lin, Q.-W. Niu, and N.H. Chua. 2006. Agrobacterium-mediated transformation of Arabidopsis thaliana using the floral dip method. Nat. Protoc. 1:641. 


\section{ELF4}
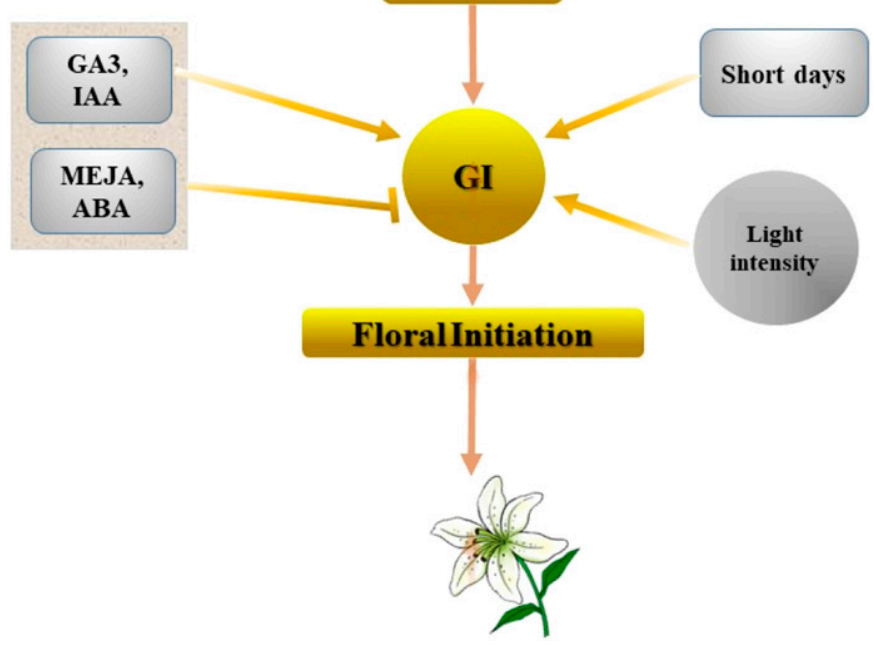

Supplemental Fig. 1. Different factors including gibberellic acid (GA3), auxin (IAA), methyl jasmonate (MEJA), and abscisic acid (ABA) affect the activity of gigentea $(G I)$ promoter. 


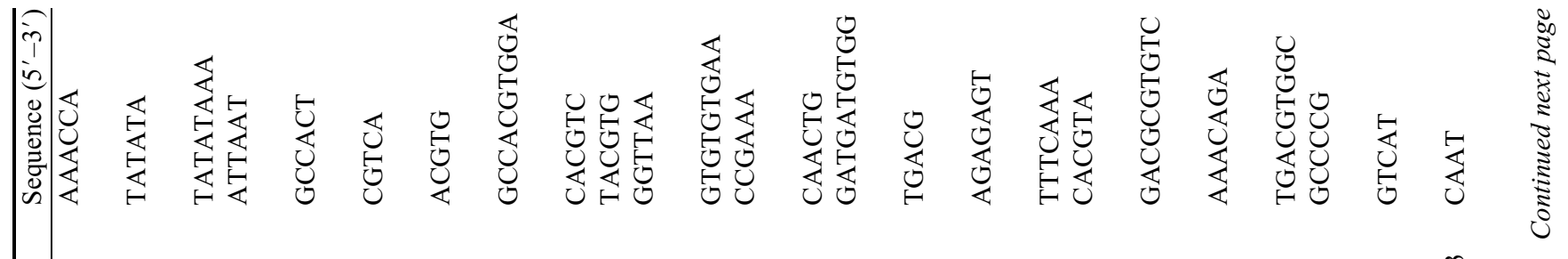

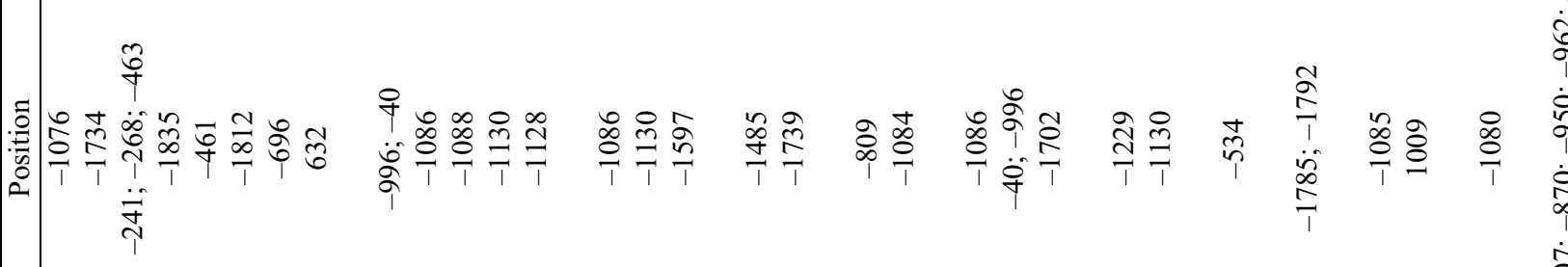

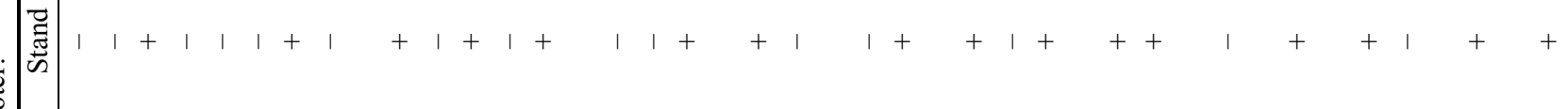

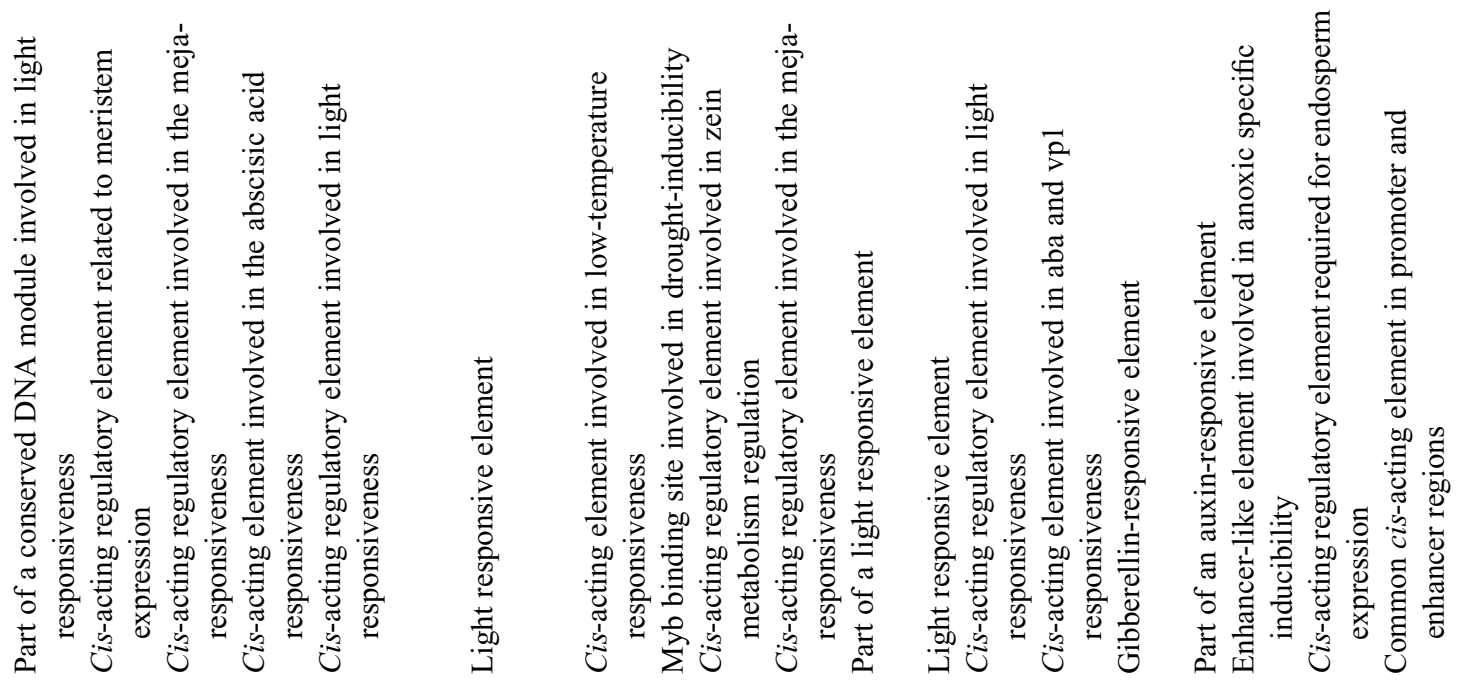

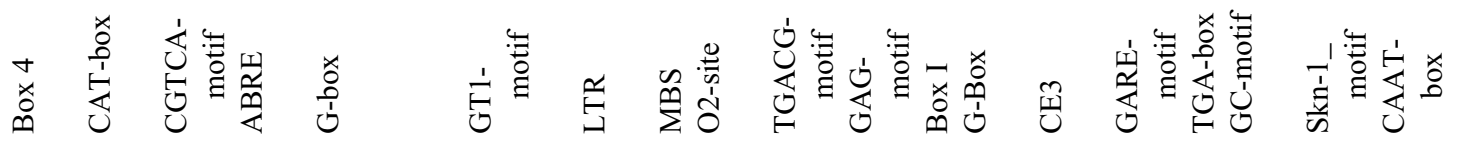




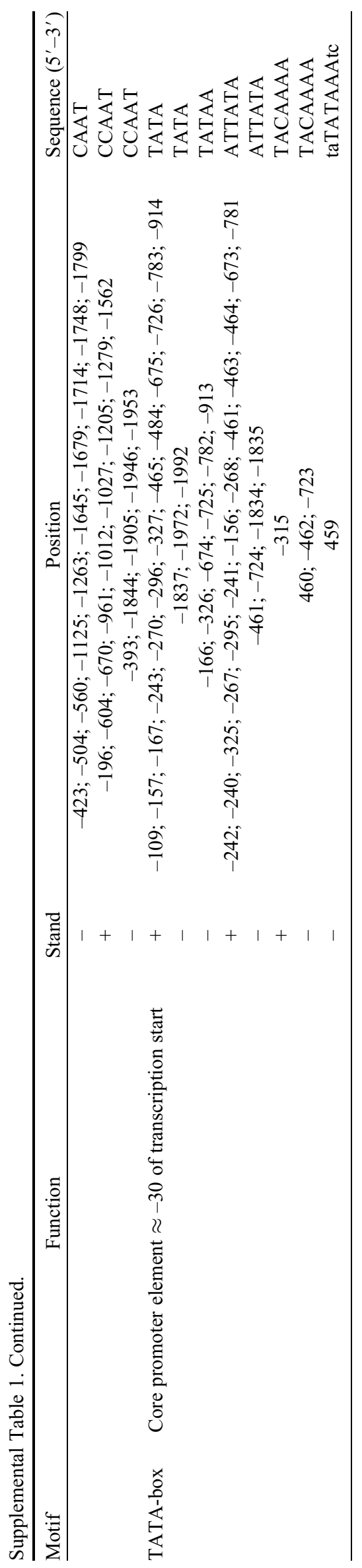

J. Amer. Soc. Hort. SCI. 145(6):1-4. 2020. 
Supplemental Table 2. Primers used in quantitative polymerase chain reaction.

\begin{tabular}{llr}
\hline Genes & Forward primer $\left(5^{\prime}\right.$ to $\left.3^{\prime}\right)$ & Reverse primer $\left(5^{\prime}\right.$ to $\left.3^{\prime}\right)$ \\
\hline GUS & TACCGTACCTCGCATTACCC & CTGTAAGTGCGCTTGCTGAG \\
Tobacco Actin & TCCTGATGGGCAAGTGATTAC & TTGTATGTGGTCTCGTGGATTC \\
Arabidopsis Actin & GCAGCATGAAGATCAAGGTGG & GTAACAAACTCACCACCACG
\end{tabular}

